\title{
Erratum: In-phase synchronization in complex oscillator networks by adaptive delayed feedback control [Phys. Rev. E 98, 042302 (2018)]
}

\author{
Viktor Novičenko (D) and Irmantas Ratas
}

(Received 16 September 2019; published 27 September 2019)

DOI: 10.1103/PhysRevE.100.039903

We would like to point out indexing errors in Eqs. (26), (27), and (31) of our paper. The correct expressions should be

$$
\begin{aligned}
& \frac{\partial V_{0}}{\partial \tau_{i}}(t)=\frac{T}{2 \pi} \sum_{j, k=1}^{N} a_{j k}\left[s_{k}(t)-s_{j}(t)\right]\left[\dot{s}_{k}(t) \frac{\partial \psi_{k \text { sync }}^{*}}{\partial \tau_{i}}-\dot{s}_{j}(t) \frac{\partial \psi_{j \text { sync }}^{*}}{\partial \tau_{i}}\right], \\
& \frac{\partial V_{0}}{\partial \tau_{i}}(t)=-\frac{K C}{\eta \varepsilon T} \sum_{j, k=1}^{N} a_{j k}\left[s_{k}(t)-s_{j}(t)\right]\left[\dot{s}_{k}(t)\left(\mathbf{L}^{\dagger}\right)_{k i}-\dot{s}_{j}(t)\left(\mathbf{L}^{\dagger}\right)_{j i}\right],
\end{aligned}
$$

and

$$
\begin{aligned}
\dot{\mathbf{x}}_{i} & =\mathbf{f}_{i}\left(\mathbf{x}_{i}, u_{i}\right)+\varepsilon \sum_{j=1}^{N} a_{i j} \mathbf{G}_{i j}\left(\mathbf{x}_{j}, \mathbf{x}_{i}\right), \\
\dot{\tau}_{i} & =-\beta q_{i}, \\
\dot{q}_{i} & =-v q_{i}-\operatorname{sgn}(K C) \sum_{j, k=1}^{N} a_{j k}\left[s_{k}-s_{j}\right]\left[\left(s_{k}-p_{k}\right)\left(\mathbf{L}^{\dagger}\right)_{k i}-\left(s_{j}-p_{j}\right)\left(\mathbf{L}^{\dagger}\right)_{j i}\right], \\
\dot{p}_{i} & =\gamma\left(s_{i}-p_{i}\right), \\
s_{i}(t) & =g\left(\mathbf{x}_{i}(t)\right), \\
u_{i}(t) & =K\left[s_{i}\left(t-\tau_{i}(t)\right)-s_{i}(t)\right],
\end{aligned}
$$

We also like to note that, in Sec. III A, the constant $\beta$ of a gradient descent method used for the case of Stuart-Landau oscillators, given five lines from the bottom on the left hand side of $\mathrm{p}$. 7 , should be $\beta=2 \times 10^{-5}$, and in Sec. III B, for the case of FitzHugh-Nagumo oscillators, given four lines from the top on the right hand side of p. 8 , it should be $\beta=3 \times 10^{-7}$.

We emphasize that these errors were misprints and do not affect the main results of the article. 